\title{
Emerging Roles of Long Non-Coding RNAs in Diabetic Foot Ulcers
}

\author{
Chengqi Yan' \\ Jing Chen' \\ Xiaofan Yang' \\ Wenqing $\mathrm{Li}^{2}$ \\ Renqun $\mathrm{MaO}^{2}$ \\ Zhenbing Chen $\mathbb{D}^{\prime}$ \\ 'Department of Hand Surgery, Union \\ Hospital, Tongji Medical College, \\ Huazhong University of Science and \\ Technology, Wuhan, 430022, People's \\ Republic of China; ${ }^{2}$ Department of Hand \\ and Foot Surgery, Huazhong University of \\ Science and Technology Union Shenzhen \\ Hospital, Shenzhen, Guangdong, People's \\ Republic of China
}

\begin{abstract}
Diabetes mellitus is one of the most widespread metabolic diseases in the world, and diabetic foot ulcer (DFU), as one of its chronic complications, not only causes a large amount of physiological and psychological pain to patients but also places a tremendous burden on the entire economy and society. Despite significant advances in knowledge on the mechanism and in the treatment of DFU, clinical practice is still not satisfactory, and our understanding of its cellular and molecular pathogenesis is far from complete. Fortunately, progress in studying the roles of long non-coding RNAs (lncRNAs), which play important regulatory roles in the expression of genes at multiple levels, suggests that we can apply them in the early diagnosis and potential targeted intervention of DFU. In this review, we briefly summarize the current knowledge regarding the functional roles and potential mechanisms of reported lncRNAs in regulating DFU.
\end{abstract}

Keywords: diabetic foot ulcer, long non-coding RNAs, diabetes mellitus, wound healing

\section{Introduction \\ Diabetic Foot Ulcer}

Diabetes mellitus is a chronic metabolic disease that is distributed worldwide. ${ }^{1}$ Diabetic patients, if left untreated, are likely to suffer from several serious acute and long-term diabetic complications, which can cause damage to multiple organs. ${ }^{2}$ Long-term exposure to hyperglycemic conditions causes macrovascular complications, along with microvascular complications, such as diabetic nephropathy, ${ }^{3}$ retinopathy, ${ }^{4}$ and diabetic foot ulcer (DFU). ${ }^{5}$ Foot ulcers in diabetic patients are referred to as lesions, which involve the loss of epithelium, sometimes extending to the dermis and even deeper layers such as bones and muscles. ${ }^{6}$

The formation of DFU can be attributed to diabetes-related peripheral neuropathy and peripheral arterial diseases. ${ }^{7,8}$ The most common form of peripheral neuropathy is distal sensorimotor polyneuropathy, where patients experience the loss of feelings and pain in the lower limbs because of distal nerve damage. ${ }^{9}$ In addition, endothelial and smooth muscle dysfunction, reduced capillary size, and progressive atherosclerosis are the reasons for microcirculation disorders and peripheral arterial disease, which occur very often in diabetic patients. ${ }^{5}$ With the progression of time, diabetes-related peripheral neuropathy and peripheral arterial diseases result in deformation and non-healing ulcers of the foot. Eventually, the dysfunctional wound healing process leads to foot deformity and the need for lower limb amputation. ${ }^{10}$

Under normal conditions, the process of wound closure involves four overlapping and coordinated stages: 1) the hemostasis stage, which stops bleeding after
Correspondence: Zhenbing Chen Department of Hand Surgery, Union Hospital, Tongji Medical College, Huazhong University of Science and Technology, Wuhan, 430022, People's Republic of China

Tel +86 I387|I 03730

Fax +862785351628

Email zbchen@hust.edu.cn 
the skin barrier is broken, in three steps: vasoconstriction, primary hemostasis, and secondary hemostasis; 2) the inflammation stage, where injured cells release various damage signals, such as chemokines and cytokines, to recruit inflammatory cells and to initiate the proliferative stage; 3$)$ the proliferation stage, where neovascularization, granulation tissue formation, re-epithelialization, and collagen deposition occur; and 4) the remodeling stage, where extracellular matrix (ECM) is periodically deposed and remodeled, and granulation tissue is reorganized by replacing type III with type I collagen. ${ }^{11}$ In DFU patients, the wound healing process slows down or even stalls owing to impairment and prolongation of the four stages. ${ }^{12,13}$

To date, there is no definitive treatment to prevent and cure DFU, and millions of patients suffer from adverse effects. ${ }^{14}$ Therefore, a better understanding of the molecular mechanisms involved in the development of diabetic complications, especially DFU, is necessary to develop better therapeutic strategies. A series of widely accepted underlying mechanisms include the excessive formation of advanced glycation end-products (AGEs), activation of protein kinase $\mathrm{C}$, increased levels of reactive oxygen species (ROS), nitric oxide blocking, and enhanced polyol pathway. ${ }^{15,16}$ In-depth research is needed, and long non-coding RNAs (lncRNAs) may provide a breakthrough point.

\section{LncRNA}

The Encyclopedia of DNA Elements project demonstrated that only approximately $2 \%$ of the genome encodes for proteins, while the remaining genome fraction encodes for thousands of non-coding RNAs, which were previously considered to be "transcriptional noise". ${ }^{17}$ A growing amount of evidence has proved that non-coding RNAs also play important roles in biological processes, such as proliferation, differentiation, apoptosis, and reprogramming. ${ }^{18-20}$ Based on the length of transcripts, IncRNAs are defined as a class of RNA molecules that are longer than 200 nucleotides, and have no or low protein-coding potential. ${ }^{21}$

The most frequently used classification method is based on the genomic location of the lncRNA and proximal protein-coding genes, and lncRNAs can be classified as intergenic, intronic, sense, antisense, and bidirectional lncRNAs. ${ }^{22,23}$ Studies have shown that IncRNAs play the role of gene regulation at transcriptional, post-transcriptional, translational, and epigenetic levels, mainly including the recruitment of transcription factors, binding to RNA polymerase II, maintaining mRNA stability, interaction with
miRNAs, regulation of DNA replication timing, and chromosome stability. ${ }^{18,24-27}$

A growing number of studies have reported that lncRNAs have great potential as predictive, diagnostic, and even therapeutic biomarkers in various types of tumors, cardiovascular diseases, and many other human diseases. ${ }^{28-30}$ For example, lncRNA Gm5524 has been reported to promote the development of diabetic nephropathy by inhibiting the expression of anti-apoptotic markers. ${ }^{31}$ In diabetic retinopathy, lncRNA H19 has been reported to be downregulated, and further study found that it may regulate endothelialmesenchymal transition through a transforming growth factor- $\beta$ (TGF- $\beta$ ) and Smad-independent mechanism. ${ }^{32}$

Similarly, many studies on expression disorders and the crucial roles of IncRNAs in DFU have been reported in recent years, which have not been reviewed yet. Thus, in this review, we summarize the current knowledge on the deregulation of IncRNAs and their functional effects on the healing process of DFU. We also discuss the potential of these lncRNAs and their upstream or downstream mediators as therapeutic targets for diabetic wound healing (Table 1).

\section{LncRNA Expression Profiling in Diabetic Foot Ulcers}

With the rapid development of the field of IncRNAs, differentially expressed lncRNAs in DFU have been studied by microarray and high-throughput deep sequencing (Table 2). Takematsu et al examined the changes in gene expression in the skin between 13 patients with type 2 diabetes and 14 non-diabetic patients. ${ }^{33}$ They found that the most upregulated genes included three IncRNAs: LINC01118, RP11545I5.3, and an unknown lncRNA. In contrast, the most downregulated genes included three lncRNAs: LINC01060, HPRT1P2, and CCNYL3. This study showed that the expression of the IncRNAs LINC01118 and LINC01060 was the most enhanced and decreased, respectively.

$\mathrm{Xu}$ et al investigated the T-lymphocyte subset in the wound surfaces of DFU. ${ }^{34}$ They found that 2142 lncRNAs were upregulated, while 1332 IncRNAs were downregulated. Then, they focused on IncRNA ENST00000411554 and revealed that the activation of the MAPK signal transduction pathway mediated by lncRNA ENST00000411554 may be one of the molecular mechanisms explaining the immune regulatory imbalance in DFU. 
Table I Functional LncRNAs in Wound Healing of Diabetic Foot Ulcers

\begin{tabular}{|c|c|c|c|c|c|}
\hline $\begin{array}{l}\text { Stage of Wound } \\
\text { Healing Process }\end{array}$ & LncRNAs & Expression & Targets & Functional Role & Ref. \\
\hline \multirow[t]{4}{*}{ Inflammation } & MALATI & Down & $\begin{array}{l}\text { HIF-I } \alpha \text {; TNF- } \alpha \text {, } \\
\text { IL-6, IL-I0 }\end{array}$ & $\begin{array}{l}\text { Has a potential pro-inflammatory role and promotes } \\
\text { angiogenesis }\end{array}$ & [66] \\
\hline & WAKMAR2 & Down & NF-kB signaling & $\begin{array}{l}\text { Has an anti-inflammatory role and promotes re-epitheli } \\
\text { alization }\end{array}$ & [67] \\
\hline & GAS5 & Up & STATI & $\begin{array}{l}\text { Has a pro-inflammatory role by inducing MI macrophage } \\
\text { phenotype }\end{array}$ & [69] \\
\hline & Lethe & Down & $\begin{array}{l}\text { p65-NF-kB } \\
\text { /NOX2 }\end{array}$ & $\begin{array}{l}\text { Has an anti-inflammatory role by eliminating increased ROS } \\
\text { production }\end{array}$ & [7I] \\
\hline \multirow[t]{9}{*}{ Proliferation } & $\mathrm{HIO}$ & - & $\begin{array}{l}\text { miR- } \mid 52-3 p / \\
\text { PTEN }\end{array}$ & $\begin{array}{l}\text { Promotes proliferation and migration, and suppresses } \\
\text { apoptosis and inflammation of fibroblasts }\end{array}$ & {$[74]$} \\
\hline & $\mathrm{HI9}$ & - & $\mathrm{EZH} 2 / \mathrm{HIF}-\mathrm{I} \alpha$ & $\begin{array}{l}\text { Promotes angiogenesis and fibroblast proliferation and } \\
\text { migration }\end{array}$ & {$[75]$} \\
\hline & $\mathrm{HI9}$ & - & PI3K/Akt & Promotes angiogenesis & {$[80]$} \\
\hline & MALATI & - & HIF-I $\alpha /$ MMP-9 & Promotes collagen deposition and the viability of fibroblasts & {$[82]$} \\
\hline & MALATI & - & miR-205-5p & Promotes angiogenesis & [84] \\
\hline & WAKMARI & Down & DNMTs/E2FI & Promotes keratinocyte migration & [88] \\
\hline & GAS5 & - & c-Myc & Promotes re-epithelialization and angiogenesis & [91] \\
\hline & TETILA & $U_{p}$ & TET2/MMP-9 & Suppresses HaCat migration & [98] \\
\hline & ANRIL & Down & $\begin{array}{l}\operatorname{miR}-\mid 8 I \mathrm{a} / \\
\text { Proxl }\end{array}$ & Promotes lymphangiogenesis in diabetic wound healing & {$[101]$} \\
\hline \multirow[t]{2}{*}{ Remodeling } & $\mathrm{HI9}$ & Up & miR-29b/FBNI & Accelerates the deposition of ECM & [103] \\
\hline & URIDS & Up & Plod I & $\begin{array}{l}\text { Decreases the deposition of collagen and the ratio of collagen } \\
\mathrm{I} / \mathrm{III}\end{array}$ & [105] \\
\hline
\end{tabular}

Table 2 The Expression Profile of LncRNAs in Diabetic Foot Ulcers

\begin{tabular}{|c|c|c|c|c|}
\hline Number & Method & Sample & Result & Ref. \\
\hline I & RNA sequencing & $\begin{array}{l}\text { Skin tissues from } 13 \text { non-diabetic } \\
\text { and } 14 \text { type } 2 \text { diabetes donors }\end{array}$ & $\begin{array}{l}5 \text { upregulated genes and } 6 \text { downregulated genes were } \\
\text { under the IncRNA category within the } 64 \text { upregulated } \\
\text { genes and } 120 \text { downregulated genes }\end{array}$ & [33] \\
\hline 2 & Microarray & $\begin{array}{l}\text { Wound skin tissues of patients } \\
\text { receiving debridement for trauma or } \\
\text { DFU associated with infection }\end{array}$ & $\begin{array}{l}\text { 2I } 42 \text { upregulated IncRNAs and I } 332 \text { downregulated } \\
\text { IncRNAs }\end{array}$ & [34] \\
\hline 3 & $\begin{array}{l}\text { ArrayQuality package and } \\
\text { the limma package to } \\
\text { analyze the GSE68I86 } \\
\text { dataset }\end{array}$ & $\begin{array}{l}3 \text { DFU samples and } 3 \text { non-diabetic } \\
\text { foot skin samples }\end{array}$ & $\begin{array}{l}58 \text { upregulated IncRNAs and } 42 \text { downregulated } \\
\text { IncRNAs }\end{array}$ & [35] \\
\hline 4 & Microarray & $\begin{array}{l}\text { Human skin fibroblasts cultured in } \\
\text { high and normal glucose }\end{array}$ & $\begin{array}{l}7 \text { upregulated IncRNAs and } 7 \text { downregulated IncRNAs } \\
\text { were associated with the angiogenesis pathway }\end{array}$ & [36] \\
\hline
\end{tabular}


In another study, Yu et al analyzed the GSE68186 dataset and, for the first time, constructed differentially expressed mRNA-mediated PPI networks and lncRNA co-expression networks using real-time PCR and gene ontology analysis. ${ }^{35}$ The 58 lncRNAs and 688 differentially expressed genes (DEGs) were associated with the upregulated IncRNAmediated co-expression network, and the 42 lncRNAs and 700 DEGs were associated with the downregulated lncRNAmediated co-expression network. These results showed that differentially expressed IncRNAs are involved in several regulatory pathways, such as the ERK1 and ERK2 cascade and secondary alcohol biosynthetic process, showing the prognostic potential of IncRNAs in DFU.

Tu et al examined the impaired angiogenesis process in diabetic wound healing and cultured human skin fibroblasts under hyperglycemic conditions in vitro to mimic a diabetic environment. ${ }^{36}$ By microarray analysis, they found that 14 angiogenesis pathway-associated lncRNAs and 22 mRNAs were differentially expressed, among which seven lncRNAs and nine mRNAs were upregulated, and seven lncRNAs and 13 mRNAs were downregulated. Then, they investigated whether lncRNA RP4-791C19.1 and CTD-2589O24.1 act as enhancers on their respective target genes: epidermal growth factor receptor (EGFR) and p21 (RAC1) activated kinase 1 (PAK1), and cis-regulate their expression according to bioinformatics predictions.

\section{Roles of LncRNAs in Diabetic Foot Ulcers}

\section{Role in Diabetic Neuropathy and Microvascular Injury}

As mentioned in the Introduction, DFU is a chronic complication that results directly from peripheral nerve dysfunction and peripheral arterial diseases associated with diabetes. ${ }^{7,8}$ In the progression of DFU, microvascular and neural damage are closely related. Blood vessels depend on normal nerve function, and nerves depend on adequate blood flow. Thus, it is necessary to review the roles of lncRNAs in diabetic neuropathy (Table 3 ) and microvascular injury.

\section{Role in Diabetic Neuropathy}

To discover a potential connection between lncRNAs and diabetic neuropathy, many researchers have applied whole-transcriptome sequencing technology to analyze the differentially expressed lncRNAs in patients or in streptozotocin (STZ)-induced diabetic rats with diabetic neuropathy. ${ }^{37-41}$ Our group conducted a microarray study to investigate the expression profiling of lncRNAs and mRNAs in dorsal root ganglia (DRG) from rats with diabetic neuropathy, and identified $983 \operatorname{lncRNAs}$ and 1357 mRNAs that were aberrantly expressed compared with control samples. ${ }^{37}$ Furthermore, lncRNA-mRNA network analysis was performed and showed dynamic interactions between the dysregulated lncRNAs and mRNAs.

It has been reported that the expression level of IncRNA NONRATT021972 was upregulated in the DRG of a diabetic rat model and in the blood of patients with type 2 diabetes. ${ }^{42-45}$ Further experiments showed that NONRATT021972 siRNA treatment increased the mechanical withdrawal threshold, the thermal withdrawal latency, and the sensory nerve conduction velocity in STZinduced diabetic rats. ${ }^{44,45}$ Mechanistically, the upregulation of P2X3, P2X7, and pro-inflammatory signals in DRG of diabetic rats could be abrogated by NONRATT021972 siRNA. $^{42,44,45}$ Moreover, Peng et al demonstrated that NONRATT021972 siRNA treatment can also reduce the hyperalgesia potentiated by the activation of ERK pathway in diabetic rats. ${ }^{44}$

$\mathrm{P} 2 \mathrm{X} 3$ and $\mathrm{P} 2 \mathrm{X} 7$ are two purinergic P2X neurotransmitter receptors, the activation of which by ATP has been shown to mediate pain hypersensitivity in diabetic neuropathy. ${ }^{46}$ Specifically, P2X3 receptors play a crucial role in pain transmission at peripheral sensory neurons, ${ }^{47}$ while P2X7 receptors are engaged both in inflammation and in neuropathic pain. ${ }^{48}$ Apart from NONRATT021972, IncRNA BC168687 and uc.48+ were positively associated with neuropathic pain scores of diabetes. Moreover, the siRNA of the two lncRNAs also downregulated P2X7 and $\mathrm{P} 2 \mathrm{X} 3$ expression in rats with diabetic neuropathy, thereby alleviating the symptoms of neuropathic pain. ${ }^{49-52}$

In a study by Liu et al, knockdown of BC168687 attenuated diabetes-induced mechanical allodynia and thermal hyperalgesia by downregulating the expression of transient receptor potential vanilloid type 1 (TRPV1). ${ }^{53}$ TRPV1 is highly correlated with a variety of pain modalities, including inflammatory pain, neuropathic pain, and pathological pain. ${ }^{54}$ Their study also reported that BC168687 siRNA treatment inhibited the upregulated p-ERK and p-p38 signaling, as well as the increased levels of tumor necrosis factor- $\alpha$ (TNF- $\alpha$ ) and interleukin- $1 \beta$ (IL$1 \beta)$ in the DRG of diabetic rats.

Both lncRNA HCG18 and nuclear enriched abundant transcript 1 (NEAT1) have been reported to contribute to the pathogenesis of diabetic neuropathy. ${ }^{55,56}$ Ren et al discovered that HCG18 promoted M1 macrophage 
Table 3 Functional LncRNAs in Diabetic Neuropathy

\begin{tabular}{|c|c|c|c|c|}
\hline LncRNA & Expression & Targets & Functional Role & Ref. \\
\hline \multirow[t]{2}{*}{ NONRATT02 1972} & \multirow[t]{2}{*}{ Up } & $\mathrm{P} 2 \times 3$ & \multirow{2}{*}{$\begin{array}{l}\text { Decreases mechanical withdrawal threshold, thermal withdrawal latency, and } \\
\text { sensory nerve conduction velocity }\end{array}$} & {$[44]$} \\
\hline & & $\mathrm{P} 2 \times 7$ & & {$[43,45]$} \\
\hline \multirow[t]{2}{*}{ uc.48+ } & \multirow[t]{2}{*}{$U_{p}$} & $\mathrm{P} 2 \times 3$ & \multirow[t]{2}{*}{ Enhances mechanical allodynia and thermal hyperalgesia } & [49] \\
\hline & & $\mathrm{P} 2 \times 7$ & & {$[50]$} \\
\hline \multirow[t]{2}{*}{ BCI 68687} & \multirow[t]{2}{*}{$U_{p}$} & $\mathrm{P} 2 \times 7$ & \multirow[t]{2}{*}{ Enhances mechanical allodynia and thermal hyperalgesia } & {$[51,52]$} \\
\hline & & TRPVI & & [53] \\
\hline HCGI8 & Up & $\begin{array}{l}\text { miR-I46a/ } \\
\text { TRAF6 }\end{array}$ & $\begin{array}{l}\text { Promotes MI macrophage polarization, and accelerates the progression of } \\
\text { diabetic peripheral neuropathy }\end{array}$ & [55] \\
\hline NEAT I & $U_{p}$ & $\begin{array}{l}\text { miR-183-5p, } \\
\text { miR-433-3p }\end{array}$ & Potential biomarkers in diabetic neuropathy & [56] \\
\hline PVTI & Down & $\mathrm{PI3K} / \mathrm{AKT}$ & Reverses the impaired nerve conduction in diabetic rats & [57] \\
\hline
\end{tabular}

polarization, which accelerates the progression of diabetic peripheral neuropathy by promoting the secretion of inflammatory factors. ${ }^{55}$ They further revealed that HCG18 regulated M1 macrophage polarization via the miR-146a/TRAF6 axis. Asadi et al found an increased expression of NEAT1, ITGA4, SESN1, and SESN3, as well as a decreased expression of miR-183-5p and miR433-3p, in patients with diabetic neuropathy. ${ }^{56}$ Their receiver operating characteristics (ROC) curve analyses indicated that these dysregulated molecules could be potential biomarkers in diabetic neuropathy.

LncRNA plasmacytoma variant translocation 1 (PVT1) has been proven to be a protective factor in diabetic neuropathy. Chen et al found that PVT1 expression was significantly downregulated in DRG of diabetic rats, and the overexpression of PVT1 reversed the impaired nerve conduction in diabetic rats. ${ }^{57}$ Their in-depth experiments demonstrated that PVT1 downregulated the expression levels of neurodegeneration-related genes (Uchl1, Sod1), while upregulating the expression levels of neurogenesis-related genes (Drd2, Notch1, and S100b). Furthermore, PVT1 activated the $\mathrm{PI} 3 \mathrm{~K} / \mathrm{AKT}$ pathway, which was blocked in diabetic rats.

\section{Role in Diabetic Microvascular Injury}

Yan et al were the first to extend the study of lncRNA into a novel research field, diabetic microvascular injury. ${ }^{58}$ They found that hyperglycemia significantly upregulated lncRNA MIAT levels in endothelial cells and diabetic retinas. Their study revealed that MIAT knockdown inhibited endothelial cell proliferation, migration, and tube formation in vitro, and alleviated pathological angiogenesis, vascular leakage, and inflammation in vivo. Since then, more and more lncRNAs have been reported to play important roles in diabetic nephropathy and retinopathy, two diabetes-related microvascular complications, and many researchers have reviewed this topic. $^{59-61}$

Hyperglycemia is the major contributor to endothelial dysfunction, which is a risk indicator for diabetic angiopathy. $\mathrm{Xu}$ et al characterized the lncRNA landscape of expression, and found that 214 lncRNAs were upregulated and 197 were downregulated in highglucose cultured human umbilical vein endothelial cells (HUVECs). ${ }^{62}$ Further research should focus on these dysregulated lncRNAs to provide novel insights into the regulatory molecules of diabetes-induced endothelial dysfunction. Hyperglycemic conditions also cause damage to endothelial progenitor cells (EPCs). EPCs are essential in angiogenesis and wound healing, but their circulating and wound level numbers are decreased in diabetes. ${ }^{63}$ In the study by $\mathrm{Li}$ et al, overexpression of lncRNA taurine upregulated gene 1 (TUG1) restored the ability of migration, invasion, and tube formation in high-glucose treated EPCs. ${ }^{64}$ In vivo, TUG1 overexpression promoted angiogenesis in a diabetic mouse ischemic limb model.

In wound healing of DFU, microvascular injury directly leads to a disorder of angiogenesis, which occurs at the beginning of the proliferation stage. LncRNAs involved in regulating the process of angiogenesis have been reviewed in 
the following subsection, "Role in wound healing of diabetic foot ulcers; Proliferation stage".

\section{Role in Wound Healing of Diabetic Foot Ulcers Inflammation Stage}

Patients with diabetic foot have a high risk of requiring leg amputation because of untreated infections. ${ }^{65}$ Jayasuriya et al investigated the role of IncRNA metastasis-associated lung adenocarcinoma transcript 1 (MALAT1) in infected DFU. ${ }^{66}$ They knocked down MALAT1, and observed that the expression of the two pro-inflammatory cytokines TNF- $\alpha$ and IL- 6 was suppressed, while the expression of the anti-inflammatory cytokine IL-10 was increased. Their preliminary research showed that MALAT1 was involved in the inflammation stage in DFU healing, which needs to be confirmed in future studies.

Herter et al revealed the function of the previously annotated but unstudied lncRNA LOC100130476 in the inflammatory phase of wound healing. ${ }^{67}$ This lncRNA was later named wound and keratinocyte migration-associated long non-coding RNA 2 (WAKMAR2). First, they found that the expression of WAKMAR2 was significantly decreased in delayed wound-edge tissues when the wound repair progression was in the inflammatory phase. Then, they revealed that knockdown of WAKMAR2 upregulated the inflammatory chemokines produced by keratinocytes and blocked the re-epithelialization phase in a human ex vivo wound model. Further research uncovered the mechanism whereby the functional loss of WAKMAR2 activated NF-kB signaling, a well-known proinflammatory pathway, ${ }^{15}$ and contributed to a hyperactive inflammatory phase and non-healing diabetic wounds.

In diabetic wounds, excessive persistence of the M1 macrophage phenotype and failure to transition to the M2 phenotype are two pathogenic features associated with delayed healing. ${ }^{68} \mathrm{Hu}$ et al focused on the inflammation stage and found that reducing the expression of lncRNA growth arrest specific 5 (GAS5) may enhance diabetic wound healing by promoting the transition of proinflammatory (M1) macrophages to anti-inflammatory (M2) macrophages. ${ }^{69}$ Their study demonstrated that GAS5 overexpression induced the expression of M1 marker genes (iNOS, IL-1 $\beta$, and TNF- $\alpha$ ) rather than M2 marker genes (Arg1 and Mrc1). A further study showed that GAS5 was a positive regulator of STAT1, which is an important inducer of the M1 macrophage phenotype. ${ }^{70}$
Excessive ROS and oxidative stress are among the reasons for clinical complications in diabetes, including diabetic impaired wound healing. ${ }^{15}$ Zgheib et al found that lncRNA Lethe was downregulated, while NOX2 was upregulated in diabetic wounds. Moreover, overexpression of Lethe decreased the formation of ROS and eliminated the upregulation of NOX2 expression in both RAW macrophage cells under hyperglycemic conditions and diabetic bone marrow-derived macrophages (BMMs). ${ }^{71}$ Their further mechanistic research revealed that Lethe exerted its function by inhibiting the translocation of p65-NF-kB to the nucleus. NOX2 is a known producer of ROS, and the p65-NF-kB complex can induce the expression of NOX2. ${ }^{72,73}$ Their study was the first to demonstrate the role of Lethe as an anti-oxidative stress IncRNA in delayed diabetic wound healing, and further studies are required to determine whether correction of Lethe expression in diabetic wounds could improve healing.

\section{Proliferation Stage}

LncRNAs with the ability to regulate the abundance or activity of other RNAs, mainly including miRNA, are called competing endogenous RNAs (ceRNAs). ${ }^{18}$ LncRNA H19 has been shown to function as a ceRNA to target miR-152-3p. ${ }^{74}$ MiR-152-3p was found to be upregulated in chronic wound tissues of patients with $\mathrm{DFU}$, and to inhibit the proliferation and migration of fibroblasts by targeting phosphatase and tensin homolog (PTEN). A study by $\mathrm{Li}$ et al proved that treatment with H19 promoted the function and suppressed the apoptosis of fibroblasts through the H19/miR-152-3p/PTEN axis, thus being a promising therapeutic strategy in diabetic wound healing. ${ }^{74}$

Similarly, Guo et al demonstrated that the enhancement of H19 expression could promote the proliferation and migration of fibroblasts in vitro and improve the coagulation function in vivo. ${ }^{75}$ In-depth research showed that H19 played a role as an enhancer for the recruitment of zeste homolog 2 (EZH2), an important histone H3K27 methyltransferase, ${ }^{76}$ to the HIF-1 $\alpha$ gene promoter zone. The recruitment of EZH 2 could promote HIF- $1 \alpha$ histone H3K4me3 methylation and increase HIF-1 $\alpha$ expression. HIF- $1 \alpha$ is an important regulator in wound healing and controls many processes, such as angiogenesis, extracellular deposition, and re-epithelialization, ${ }^{77}$ and the upregulation of HIF-1 $\alpha$ promotes diabetic wound healing.

Chronic diabetic wounds can be attributed to defects in the angiogenesis phase of the healing process. ${ }^{6} \mathrm{H} 19$ has 
been proven to be important in the process of angiogenesis. $^{78,79}$ In their preliminary experiment, Tao et al transfected HEK293 (human embryonic kidney 293) cells with an H19-overexpressing lentiviral vector and harvested the extracellular vesicles (EVs) of this cell line, ${ }^{80}$ which stably expressed H19 and were named H19EMNVs (extracellular vesicle-mimetic nanovesicles). EVs are popular owing to their function of treating and diagnosing diseases in various fields, including diabetic wound healing. ${ }^{81}$ Then, they proved that H19-EMNVs could improve the proliferation, migration, and vessel formation of endothelial cells, thus promoting angiogenesis in the wound healing of DFU.

MALAT1 can also regulate HIF-1 $\alpha$ expression to help patients heal their impaired diabetic wounds. ${ }^{82}$ Liu et al found that enhancement of MALAT1 and the HIF-1 $\alpha$ signaling pathway could improve fibroblast activation, promote collagen expression, and accelerate wound healing in a diabetic mouse model. Their study shed lights on the upregulation of MALAT1 as a possible strategy to treat delayed diabetic wounds. ${ }^{82}$

Mesenchymal stem cells (MSCs) have multipotent differential potential and are used to promote tissue repair. ${ }^{83}$ Zhu et al overexpressed MALAT1 in MSCs. ${ }^{84}$ When the modified MSCs were transplanted into wound tissues in STZ-induced diabetic mice, higher vessel density was detected, which reflected promotion of the angiogenesis phase. Further research showed that MALAT1 played its role by downregulating the expression of miR-205-5p. Their study put forward new ideas for using lncRNAs, which can function as a sponge RNA, to optimize the MSC therapeutic strategy to treat DFU.

Re-epithelialization is an essential part of the proliferation stage in wound healing, and it is mainly attributed to keratinocyte migration. ${ }^{11,85}$ In many chronic wounds, including DFU, keratinocyte migration and the process of reepithelialization are impaired. ${ }^{13,86,87} \mathrm{Li}$ et al found that knockdown and overexpression of IncRNA WAKMAR1 reduced and increased, respectively, the motility of keratinocytes and re-epithelialization of human ex vivo skin wounds. ${ }^{88}$ Mechanistically, WAKMAR1 regulated a network of genes that are important for cell migration by enhancing the expression of E2F transcription factor 1 (E2F1). E2F1 is a transcription factor (TF) functioning to direct many biological activities, such as cell proliferation and migration. ${ }^{89,90}$ This line of evidence demonstrated that WAKMAR1 is a critical IncRNA in promoting keratinocyte migration, a deficiency of which may impair wound re-epithelialization.
Sawaya et al revealed that mevastatin, one of the common statin drugs, may be a novel option for the treatment of chronic diabetic wounds by promoting epithelialization and angiogenesis. $^{91}$ They found that topical application of mevastatin upregulated GAS5 expression, thus downregulating c-Myc expression in DFU, which has been proven to be a wound healing inhibitor. ${ }^{92,93}$ Their study demonstrated that GAS5 may have a positive impact in the healing of DFU, while $\mathrm{Hu}$ et al claimed that a prolonged inflammation stage may be attributed to GAS5 overexpression. ${ }^{69}$ Therefore, more research is needed to explore the mechanism and function of GAS5 in wound healing of DFU.

Excessive activation of matrix metalloproteinase-9 (MMP-9) is one factor that leads to delayed diabetic wound healing. ${ }^{94,95}$ LncRNAs can play their roles at the epigenetic level, which includes histone and DNA methylation. The process of DNA demethylation requires enzymatic activity of multiple proteins, such as DNA demethylase TET2. ${ }^{96,97}$ Zhou et al found that knockdown of TET2-interacting IncRNA (TETILA) reduced TET2 protein expression, TET2 activity, and MMP-9 transcription, while promoting HaCat migration and reversing AGE-induced wound healing deficits. ${ }^{98}$ Further research uncovered the molecular mechanism: TETILA served as a homing signal to recruit TET2, thus inducing promoter demethylation and promoting the expression of MMP-9 to disrupt the process of diabetic wound healing.

Lymphangiogenesis occurs in adult tissues during wound healing, and lymphatic vessels play important roles in this process. ${ }^{99}$ Evidence has shown that impaired lymphangiogenesis was implicated in non-healing wounds. $^{100}$ He et al found that overexpression of lncRNA antisense non-coding RNA in the INK4 locus (ANRIL) or suppression of miR-181a could rescue the apoptosis, impaired migration, and decreased tubeformation capacity in high-glucose-treated lymphatic endothelial cells. ${ }^{101}$ They revealed that ANRIL could sponge and suppress the expression of miR-181a, thus attenuating the downregulation of Prox 1 and restoring the slow lymphangiogenesis in diabetic wound healing. Prox1 is a typical lymphatic endothelial marker and a target gene of miR-181a. ${ }^{102}$ Their study uncovered the significant function of ANRIL in lymphangiogenesis, providing a promising therapeutic target to treat DFU.

\section{Remodeling Stage}

Li et al detected that the expression of H19 and Fibrillin 1 (FBN1) was higher and the expression of miR-29b was 
lower in the wound tissues of DFU patients. ${ }^{103} \mathrm{FBN} 1$ is a major component of microfibrils in the ECM and is one of the target genes of miR-29b. ${ }^{104}$ They demonstrated that the proliferation and migration of fibroblasts extracted from DFU patients were promoted, while the apoptosis of the cell line was suppressed, when H19 and FBN1 were overexpressed and miR-29b was silenced. The following mechanism underlies these phenomena: H19 competitively binds to miR-29b, thus rescuing the downregulation of FBN1. In this way, H19 accelerated the remodeling process of wound healing in their DFU mouse model.

$\mathrm{Hu}$ et al annotated and characterized a novel lncRNA, MRAK052872, which was significantly upregulated in the fibroblasts of the dermal layer in diabetic skin tissue. ${ }^{105}$ Therefore, this IncRNA was later named URIDS (UpRegulated in Diabetic Skin). They further studied its function by knocking down the expression of URIDS and found that the migration of AGE-treated fibroblasts was significantly increased. In an established diabetic wound rat model, knockdown of URIDS increased the deposition of collagen and the ratio of collagen I/III. Later, they identified procollagen-lysine, 2-oxoglutarate 5-dioxygenase 1
(Plod1) as a URIDS-targeting protein. Plod1 encodes lysyl hydroxylase 1 , which is a crucial enzyme that catalyzes the formation of hydroxylysine in the process of collagen synthesis, and is responsible for ECM remodeling and stabilization. ${ }^{106}$ Their study collectively revealed URIDS as a potential therapeutic target for the pathological process.

\section{Conclusion}

Refractory DFU is a chronic diabetes complication that lacks effective treatment measures, leading to an intractable clinical challenge and a serious public health issue. Although many researchers have explored the specific molecular biological mechanism of this disease in multiple physiological and pathological processes, the understanding of the roles of IncRNAs in the occurrence, development, and progression of DFU is still in its infancy. Based on the evidence reviewed in this article, aberrant expression of lncRNAs disrupts the orderly process of wound healing in DFU, and the IncRNAs exert their effect on gene regulation through sponging to miRNAs (H19, MALAT1, etc.), recruiting epigenetic related enzymes (WAKMAR1, TETILA, H19, etc.), regulating protein stability (Lethe, Plod1, etc.), and other processes (Figure 1).

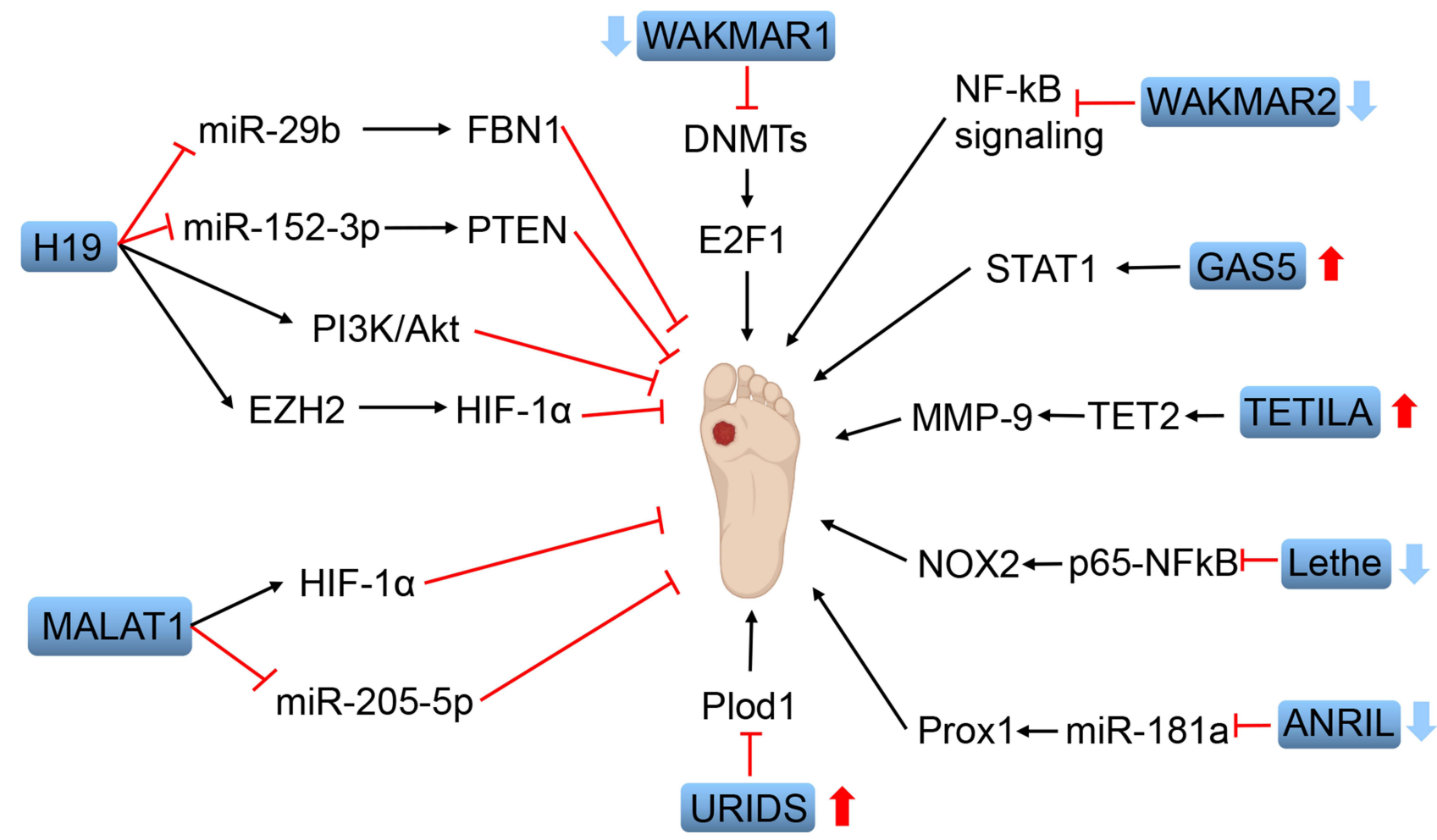

Figure I Schematic diagram of functional IncRNAs in wound healing of diabetic foot ulcers. Dysregulation of these IncRNAs is related to the pathogenesis of diabetic foot ulcers. Red arrows indicate the upregulated IncRNAs (GAS5, TETILA, URIDS) and blue arrows indicate the downregulated IncRNAs (WAKMARI, WAKMAR2, Lethe, ANRIL). The application of $\mathrm{HI} 9$ and MALATI can accelerate the wound healing of diabetic foot ulcers through different mechanisms. 
The annotated lncRNAs discussed in this review are just the tip of the iceberg, and many questions remain unanswered. Therefore, we suggest that future studies could pay attention to the following questions. First, significant efforts should be made to clarify the function of unannotated IncRNAs related to DFU, which could provide a more comprehensive insight into the molecular basis of the disease and lead to new promising therapeutics and diagnostics. Moreover, existing research on wound healing focuses on the proliferation stage, including the reepithelialization and neovascularization process. More research should focus on other wound healing stages, such as the inflammation and remodeling stages. Smallmolecule targeted therapies have been carried out in many disease areas, and further research should be conducted to explore nucleic acid-based and particularly lncRNA-based therapeutics in DFU. Furthermore, many challenges exist in transferring the significant results of cell and animal experiments to the final clinical applications. For example, differences between in vitro and in vivo experiments are unpredictable. Therefore, detailed functional verification in various models of DFU is indispensable for the confirmation of lncRNAs with potential for clinical application.

\section{Consent for Publication}

All the authors give their consent for publication.

\section{Acknowledgment}

We kindly thank the National Natural Science Foundation of China for the funding.

\section{Funding}

This work was supported by the National Natural Science Foundation of China (81974289 and 81772094).

\section{Disclosure}

The authors declare that they have no competing interests in this work.

\section{References}

1. Saeedi P, Petersohn I, Salpea P, et al. Global and regional diabetes prevalence estimates for 2019 and projections for 2030 and 2045: results from the International Diabetes Federation Diabetes Atlas, 9 (th) edition. Diabetes Res Clin Pract. 2019;157:107843. doi:10.1016/j. diabres.2019.107843

2. Forbes JM, Cooper ME. Mechanisms of diabetic complications. Physiol Rev. 2013;93(1):137-188. doi:10.1152/physrev.00 045.2011
3. Tervaert TWC, Mooyaart AL, Amann K, et al. Pathologic classification of diabetic nephropathy. J Am Soc Nephrol. 2010;21 (4):556-563. doi:10.1681/ASN.2010010010

4. Rosenberg JB, Tsui I. Screening for diabetic retinopathy. $N$ Engl $J$ Med. 2017;376(16):1587-1588. doi:10.1056/NEJMe1701820

5. Grennan D. Diabetic foot ulcers. JAMA. 2019;321(1):114. doi:10.1001/jama.2018.18323

6. Falanga V. Wound healing and its impairment in the diabetic foot. Lancet (London, England). 2005;366(9498):1736-1743. doi:10.1016/S0140-6736(05)67700-8

7. Edmonds ME, Foster AVM. Diabetic foot ulcers. BMJ. 2006;332 (7538):407-410. doi:10.1136/bmj.332.7538.407

8. Parmet S, Glass TJ, Glass RM. JAMA patient page. Diabetic foot ulcers. JAMA. 2005;293(2):260. doi:10.1001/jama.293.2.260

9. Feldman EL, Callaghan BC, Pop-Busui R, et al. Diabetic neuropathy. Nat Rev Dis Primers. 2019;5(1):42. doi:10.1038/ s41572-019-0097-9

10. Mishra SC, Chhatbar KC, Kashikar A, et al. Diabetic foot. $B M J$. 2017;359:j5064. doi:10.1136/bmj.j5064

11. Rodrigues M, Kosaric N, Bonham CA, et al. Wound healing: a cellular perspective. Physiol Rev. 2019;99(1):665-706. doi:10.1152/physrev.00067.2017

12. Zubair M, Ahmad J. Role of growth factors and cytokines in diabetic foot ulcer healing: a detailed review. Rev Endocr Metab Disord. 2019;20(2):207-217. doi:10.1007/s11154-019-09492-1

13. Davis FM, Kimball A, Boniakowski A, et al. Dysfunctional wound healing in diabetic foot ulcers: new crossroads. Curr Diab Rep. 2018;18(1):2. doi:10.1007/s11892-018-0970-z

14. Cavanagh PR, Lipsky BA, Bradbury AW, et al. Treatment for diabetic foot ulcers. Lancet (London, England). 2005;366 (9498):1725-1735. doi:10.1016/S0140-6736(05)67699-4

15. Blakytny R, Jude E. The molecular biology of chronic wounds and delayed healing in diabetes. Diabet Med. 2006;23 (6):594-608. doi:10.1111/j.1464-5491.2006.01773.x

16. Gary Sibbald R, Woo KY. The biology of chronic foot ulcers in persons with diabetes. Diabetes Metab Res Rev. 2008;24(Suppl 1):S25-30. doi:10.1002/dmrr.847

17. Ecker JR, Bickmore WA, Barroso I, et al. Genomics: ENCODE explained. Nature. 2012;489(7414):52-55. doi:10.1038/489052a

18. Kopp F, Mendell JT. Functional classification and experimental dissection of long noncoding RNAs. Cell. 2018;172(3):393-407. doi:10.1016/j.cell.2018.01.011

19. Ransohoff JD, Wei Y, Khavari PA. The functions and unique features of long intergenic non-coding RNA. Nat Rev Mol Cell Biol. 2018;19(3):143-157. doi:10.1038/nrm.2017.104

20. Palazzo AF, Koonin EV. Functional long non-coding RNAs evolve from junk transcripts. Cell. 2020;183(5):1151-1161. doi:10.1016/j.cell.2020.09.047

21. Wu H, Yang L, Chen L. The diversity of long noncoding RNAs and their generation. Trends Genet. 2017;33(8):540-552. doi:10.1016/j.tig.2017.05.004

22. Rinn JL, Chang HY. Genome regulation by long noncoding RNAs. Апnu Rev Biochem. 2012;81:145-166. doi:10.1146/ annurev-biochem-051410-092902

23. Ma L, Bajic VB, Zhang Z. On the classification of long non-coding RNAs. RNA Biol. 2013;10(6):925-933. doi:10.4161/rna.24604

24. Fernandes JCR, Acuña SM, Aoki JI, et al. Long non-coding RNAs in the regulation of gene expression: physiology and disease. Noncoding RNA. 2019;5(1):17. doi:10.3390/ncrna5010017

25. Delás MJ, Hannon GJ. LncRNAs in development and disease: from functions to mechanisms. Open Biol. 2017;7(7):170121. doi:10.1098/rsob.170121

26. Beermann J, Piccoli M, Viereck J, et al. Non-coding RNAs in development and disease: background, mechanisms, and therapeutic approaches. Physiol Rev. 2016;96(4):1297-1325. doi:10.1152/physrev.00041.2015 
27. Statello L, Guo CJ, Chen LL, et al. Gene regulation by long non-coding RNAs and its biological functions. Nat Rev Mol Cell Biol. 2021;22(2):96-118. doi:10.1038/s41580-020-00315-9

28. Zhou R, Chen KK, Zhang J, et al. The decade of exosomal long RNA species: an emerging cancer antagonist. Mol Cancer. 2018;17(1):75. doi:10.1186/s12943-018-0823-z

29. Sallam T, Sandhu J, Tontonoz P. Long noncoding RNA discovery in cardiovascular disease. Circ Res. 2018;122(1):155-166. doi:10.1161/CIRCRESAHA.117.311802

30. Matsui M, Corey DR. Non-coding RNAs as drug targets. Nat Rev Drug Discov. 2017;16(3):167-179. doi:10.1038/nrd.2016.117

31. Feng Y, Chen S, Xu J, et al. Dysregulation of IncRNAs GM5524 and GM15645 involved in high-glucose-induced podocyte apoptosis and autophagy in diabetic nephropathy. Mol Med Rep. 2018;18(4):3657-3664. doi:10.3892/mmr.2018.9412

32. Thomas AA, Biswas S, Feng B, et al. LncRNA H19 prevents endothelial-mesenchymal transition in diabetic retinopathy. Diabetologia. 2019;62(3):517-530. doi:10.1007/s00125-0184797-6

33. Takematsu E, Spencer A, Auster J, et al. Genome wide analysis of gene expression changes in skin from patients with type 2 diabetes. PLoS One. 2020;15(2):e0225267. doi:10.1371/journal. pone. 0225267

34. Xu S, Weng X, Wang Y, et al. Screening and preliminary validation of $\mathrm{T}$ lymphocyte immunoregulationassociated long noncoding RNAs in diabetic foot ulcers. Mol Med Rep. 2019;19 (3):2368-2376. doi:10.3892/mmr.2019.9877

35. Yu P, Guo J, Li J, et al. Co-expression network analysis revealing the key lncRNAs in diabetic foot ulcers. Arch Med Sci. 2019;15 (5):1123-1132. doi:10.5114/aoms.2019.84699

36. Tu L, Huang Q, Hu Y, et al. Detection and analysis of angiogenesis pathway-associated lncRNA expression profiles in human skin fibroblasts under high-glucose conditions. Mol Med Rep. 2020;22(3):2283-2290. doi:10.3892/mmr.2020.11333

37. Guo G, Ren S, Kang Y, et al. Microarray analyses of lncRNAs and mRNAs expression profiling associated with diabetic peripheral neuropathy in rats. $J$ Cell Biochem. 2019;120 (9):15347-15359. doi:10.1002/jcb.28802

38. Du H, Liu Z, Tan X, et al. Identification of the genome-wide expression patterns of long non-coding RNAs and mRNAs in mice with streptozotocin-induced diabetic neuropathic pain. Neuroscience. 2019;402:90-103. doi:10.1016/j. neuroscience.2018.12.040

39. Luo L, Ji L, Cai J, et al. Microarray analysis of long noncoding RNAs in female diabetic peripheral neuropathy patients. Cell Physiol Biochem. 2018;46(3):1209-1217. doi:10.1159/000489071

40. Fachrul M, Utomo DH, Parikesit AA. LncRNA-based study of epigenetic regulations in diabetic peripheral neuropathy. In Silico Pharmacol. 2018;6(1):7. doi:10.1007/s40203-018-0042-8

41. Wang $\mathrm{C}, \mathrm{Xu} \mathrm{X}$, Chen $\mathrm{J}$, et al. The construction and analysis of IncRNA-miRNA-mRNA competing endogenous RNA network of schwann cells in diabetic peripheral neuropathy. Front Bioeng Biotechnol. 2020;8:490. doi:10.3389/fbioe.2020.00490

42. Yu W, Zhao G, Cao R, et al. LncRNA NONRATT021972 was associated with neuropathic pain scoring in patients with type 2 diabetes. Behav Neurol. 2017;2017:2941297. doi:10.1155/2017/ 2941297

43. Xu H, He L, Liu C, et al. LncRNA NONRATT021972 siRNA attenuates $\mathrm{P} 2 \mathrm{X} 7$ receptor expression and inflammatory cytokine production induced by combined high glucose and free fatty acids in PC12 cells. Purinergic Signal. 2016;12(2):259-268. doi:10.1007/s11302-016-9500-0

44. Peng H, Zou L, Xie J, et al. IncRNA NONRATT021972 siRNA decreases diabetic neuropathic pain mediated by the P2X3 receptor in dorsal root ganglia. Mol Neurobiol. 2017;54(1):511-523. doi:10.1007/s12035-015-9632-1
45. Liu S, Zou L, Xie J, et al. LncRNA NONRATT021972 siRNA regulates neuropathic pain behaviors in type 2 diabetic rats through the P2X7 receptor in dorsal root ganglia. Mol Brain. 2016;9:44. doi:10.1186/s13041-016-0226-2

46. Bernier L, Ase AR, Séguéla P. P2X receptor channels in chronic pain pathways. $B r \quad J$ Pharmacol. 2018;175(12):2219-2230. doi:10.1111/bph.13957

47. Inoue $K$, Tsuda $M$. Nociceptive signaling mediated by $P 2 X 3$, P2X4 and P2X7 receptors. Biochem Pharmacol. 2020;114309. doi:10.1016/j.bcp.2020.114309

48. Zhang W, Zhu Z, Liu Z. The role and pharmacological properties of the P2X7 receptor in neuropathic pain. Brain Res Bull. 2020;155:19-28. doi:10.1016/j.brainresbull.2019.11.006

49. Wang $\mathrm{S}, \mathrm{Xu} \mathrm{H}, \mathrm{Zou} \mathrm{L}$, et al. LncRNA uc.48+ is involved in diabetic neuropathic pain mediated by the $\mathrm{P} 2 \mathrm{X} 3$ receptor in the dorsal root ganglia. Purinergic Signal. 2016;12(1):139-148. doi:10.1007/s11302-015-9488-x

50. Wu B, Zhang C, Zou L, et al. LncRNA uc. $48+$ siRNA improved diabetic sympathetic neuropathy in type 2 diabetic rats mediated by P2X7 receptor in SCG. Auton Neurosci. 2016;197:14-18. doi:10.1016/j.autneu.2016.04.001

51. Liu C, Tao J, Wu H, et al. Effects of IncRNA BC168687 siRNA on diabetic neuropathic pain mediated by P2X (7) receptor on SGCs in DRG of rats. Biomed Res Int. 2017;2017:7831251. doi:10.1155/2017/7831251

52. Liu C, Deng Z, Du E, et al. Long non-coding RNA BC168687 small interfering RNA reduces high glucose and high free fatty acid-induced expression of P2X7 receptors in satellite glial cells. Mol Med Rep. 2018;17(4):5851-5859. doi:10.3892/mmr.2018.8601

53. Liu C, Li C, Deng Z, et al. Long Non-coding RNA BC168687 is involved in TRPV1-mediated diabetic neuropathic pain in rats. Neuroscience. 2018;374:214-222. doi:10.1016/j. neuroscience.2018.01.049

54. Moore C, Gupta R, Jordt S, et al. Regulation of pain and itch by TRP channels. Neurosci Bull. 2018;34(1):120-142. doi:10.1007/ s12264-017-0200-8

55. Ren $\mathrm{W}, \mathrm{Xi}$ G, Li X, et al. Long non-coding RNA HCG18 promotes M1 macrophage polarization through regulating the miR-146a/TRAF6 axis, facilitating the progression of diabetic peripheral neuropathy. Mol Cell Biochem. 2021;476(1):471-482. doi:10.1007/s11010-020-03923-3

56. Asadi G, Rezaei Varmaziar F, Karimi M, et al. Determination of the transcriptional level of long non-coding RNA NEAT-1, downstream target microRNAs, and genes targeted by microRNAs in diabetic neuropathy patients. Immunol Lett. 2021;232:20-26. doi:10.1016/j.imlet.2021.01.007

57. Chen L, Gong H, Xu L. PVT1 protects diabetic peripheral neuropathy via PI3K/AKT pathway. Eur Rev Med Pharmacol Sci. 2018;22(20):6905-6911. doi:10.26355/eurrev_201810_16160

58. Yan B, Yao J, Liu J, et al. LncRNA-MIAT regulates microvascular dysfunction by functioning as a competing endogenous RNA. Circ Res. 2015;116(7):1143-1156. doi:10.1161/CIRCRESAHA.116.305510

59. Raut SK, Khullar M. The big entity of new RNA world: long non-coding RNAs in microvascular complications of diabetes. Front Endocrinol (Lausanne). 2018;9:300. doi:10.3389/ fendo.2018.00300

60. Biswas S, Sarabusky M, Chakrabarti S. Diabetic retinopathy, IncRNAs, and Inflammation: a dynamic, interconnected network. J Clin Med. 2019;8(7):1033. doi:10.3390/jcm8071033

61. Guo J, Liu Z, Gong R. Long noncoding RNA: an emerging player in diabetes and diabetic kidney disease. Clin Sci (Lond). 2019;133 (12):1321-1339. doi:10.1042/CS20190372

62. Xu E, Hu X, Li X, et al. Analysis of long non-coding RNA expression profiles in high-glucose treated vascular endothelial cells. BMC Endocr Disord. 2020;20(1):107. doi:10.1186/s12902020-00593-6 
63. Gallagher KA, Liu Z, Xiao M, et al. Diabetic impairments in NO-mediated endothelial progenitor cell mobilization and homing are reversed by hyperoxia and SDF-1 alpha. J Clin Invest. 2007;117(5):1249-1259. doi:10.1172/JCI29710

64. Li Y, Zhi K, Han S, et al. TUG1 enhances high glucose-impaired endothelial progenitor cell function via miR-29c-3p/PDGF-BB /Wnt signaling. Stem Cell Res Ther. 2020;11(1):441. doi:10.1186/s13287-020-01958-3

65. Matos M, Mendes R, Silva AB, et al. Physical activity and exercise on diabetic foot related outcomes: a systematic review. Diabetes Res Clin Pract. 2018;139:81-90. doi:10.1016/j. diabres.2018.02.020

66. Jayasuriya R, Dhamodharan U, Karan AN, et al. Role of Nrf2 in MALAT1/HIF-1alpha loop on the regulation of angiogenesis in diabetic foot ulcer. Free Radic Biol Med. 2020;156:168-175. doi:10.1016/j.freeradbiomed.2020.05.018

67. Herter EK, Li D, Toma MA, et al. WAKMAR2, a long noncoding RNA downregulated in human chronic wounds, modulates keratinocyte motility and production of inflammatory chemokines. $J$ Invest Dermatol. 2019;139(6):1373-1384. doi:10.1016/j. jid.2018.11.033

68. Kim SY, Nair MG. Macrophages in wound healing: activation and plasticity. Immunol Cell Biol. 2019;97(3):258-267. doi:10.1111/imcb. 12236

69. Hu J, Zhang L, Liechty C, et al. Long noncoding RNA GAS5 regulates macrophage polarization and diabetic wound healing. $J$ Invest Dermatol. 2020;140(8):1629-1638. doi:10.1016/j. jid.2019.12.030

70. Ohmori Y, Hamilton TA. Requirement for STAT1 in LPS-induced gene expression in macrophages. J Leukoc Biol. 2001;69 (4):598-604

71. Zgheib C, Hodges MM, Hu J, et al. Long non-coding RNA Lethe regulates hyperglycemia-induced reactive oxygen species production in macrophages. PLoS One. 2017;12(5):e0177453. doi:10.1371/journal.pone.0177453

72. Rojas M, Zhang W, Xu Z, et al. Requirement of NOX2 expression in both retina and bone marrow for diabetes-induced retinal vascular injury. PLoS One. 2013;8(12):e84357. doi:10.1371/journal.pone. 0084357

73. Anrather J, Racchumi G, Iadecola C. NF-kappaB regulates phagocytic NADPH oxidase by inducing the expression of gp91phox. $J$ Biol Chem. 2006;281(9):5657-5667. doi:10.1074/jbc. M506172200

74. Li B, Luan S, Chen J, et al. The MSC-derived exosomal lncRNA H19 promotes wound healing in diabetic foot ulcers by upregulating PTEN via microRNA-152-3p. Mol Ther Nucleic Acids. 2020;19:814-826. doi:10.1016/j.omtn.2019.11.034

75. Guo J, Yin L, Chen Y, et al. Autologous blood transfusion augments impaired wound healing in diabetic mice by enhancing lncRNA H19 expression via the HIF-1 $\alpha$ signaling pathway. Cell Commun Signal. 2018;16(1):84. doi:10.1186/s12964-018-0290-6

76. Viré E, Brenner C, Deplus R, et al. The polycomb group protein EZH2 directly controls DNA methylation. Nature. 2006;439 (7078):871-874. doi:10.1038/nature04431

77. Hong $\mathrm{WX}, \mathrm{Hu} \mathrm{MS}$, Esquivel $\mathrm{M}$, et al. The role of hypoxia-inducible factor in wound healing. Adv Wound Care (New Rochelle). 2014;3(5):390-399. doi:10.1089/ wound.2013.0520

78. Yang Y, Tang F, Wei F, et al. Silencing of long non-coding RNA H19 downregulates CTCF to protect against atherosclerosis by upregulating PKD1 expression in ApoE knockout mice. Aging (Albany NY). 2019;11(22):10016-10030. doi:10.18632/ aging. 102388

79. Shi X, Wei Y, Li H, et al. Long non-coding RNA H19 in atherosclerosis: what role? Mol Med. 2020;26(1):72. doi:10.1186/ s10020-020-00196-w
80. Tao S, Rui B, Wang Q, et al. Extracellular vesicle-mimetic nanovesicles transport LncRNA-H19 as competing endogenous RNA for the treatment of diabetic wounds. Drug Deliv. 2018;25 (1):241-255. doi:10.1080/10717544.2018.1425774

81. Ren S, Chen J, Duscher D, et al. Microvesicles from human adipose stem cells promote wound healing by optimizing cellular functions via AKT and ERK signaling pathways. Stem Cell Res Ther. 2019;10(1):47. doi:10.1186/s13287-019-1152-x

82. Liu X, Duan L, Chen Y, et al. LncRNA MALAT1 accelerates wound healing of diabetic mice transfused with modified autologous blood via the HIF-1 $\alpha$ signaling pathway. Mol Ther Nucleic Acids. 2019;17:504-515. doi:10.1016/j.omtn.2019.05.020

83. Casiraghi F, Perico N, Cortinovis M, et al. Mesenchymal stromal cells in renal transplantation: opportunities and challenges. Nat Rev Nephrol. 2016;12(4):241-253. doi:10.1038/nrneph.2016.7

84. Zhu L, Zhong Q, Yang T, et al. Improved therapeutic effects on diabetic foot by human mesenchymal stem cells expressing MALAT1 as a sponge for microRNA-205-5p. Aging (Albany NY). 2019;11(24):12236-12245. doi:10.18632/aging.102562

85. Dekoninck S, Blanpain C. Stem cell dynamics, migration and plasticity during wound healing. Nat Cell Biol. 2019;21 (1):18-24. doi:10.1038/s41556-018-0237-6

86. Pastar I, Stojadinovic O, Yin NC, et al. Epithelialization in wound healing: a comprehensive review. Adv Wound Care (New Rochelle). 2014;3(7):445-464. doi:10.1089/wound.2013.0473

87. Keyes BE, Liu S, Asare A, et al. Impaired epidermal to dendritic T Cell signaling slows wound repair in aged akin. Cell. 2016;167 (5):1323-1338.e14. doi:10.1016/j.cell.2016.10.052

88. Li D, Kular L, Vij M, et al. Human skin long noncoding RNA WAKMAR1 regulates wound healing by enhancing keratinocyte migration. Proc Natl Acad Sci U S A. 2019;116(19):9443-9452. doi:10.1073/pnas.1814097116

89. Ertosun MG, Hapil FZ, Osman Nidai O. E2F1 transcription factor and its impact on growth factor and cytokine signaling. Cytokine Growth Factor Rev. 2016;31:17-25. doi:10.1016/j. cytogfr.2016.02.001

90. Benevolenskaya EV, Frolov MV. Emerging links between E2F control and mitochondrial function. Cancer Res. 2015;75 (4):619-623. doi:10.1158/0008-5472.CAN-14-2173

91. Sawaya AP, Pastar I, Stojadinovic O, et al. Topical mevastatin promotes wound healing by inhibiting the transcription factor c-Myc via the glucocorticoid receptor and the long non-coding RNA Gas5. J Biol Chem. 2018;293(4):1439-1449. doi:10.1074/ jbc.M117.811240

92. Stojadinovic O, Brem H, Vouthounis C, et al. Molecular pathogenesis of chronic wounds: the role of beta-catenin and c-myc in the inhibition of epithelialization and wound healing. $\mathrm{Am}$ $J$ Pathol. 2005;167(1):59-69. doi:10.1016/s0002-9440(10) 62953-7

93. Shi Y, Shu B, Yang R, et al. Wnt and Notch signaling pathway involved in wound healing by targeting c-Myc and Hes 1 separately. Stem Cell Res Ther. 2015;6(1):120. doi:10.1186/ s13287-015-0103-4

94. Liu $\mathrm{Y}$, Min D, Bolton $\mathrm{T}$, et al. Increased matrix metalloproteinase-9 predicts poor wound healing in diabetic foot ulcers. Diabetes Care. 2009;32(1):117-119. doi:10.2337/dc080763

95. Patel S, Srivastava S, Singh MR, et al. Mechanistic insight into diabetic wounds: pathogenesis, molecular targets and treatment strategies to pace wound healing. Biomed Pharmacother. 2019;112:108615. doi:10.1016/j.biopha.2019.108615

96. Kiefer JC. Epigenetics in development. Dev Dyn. 2007;236 (4):1144-1156. doi:10.1002/dvdy.21094

97. Weichenhan D, Plass C. The evolving epigenome. Hum Mol Genet. 2013;22(R1):R1-6. doi:10.1093/hmg/ddt348 
98. Zhou L, Ren M, Zeng T, et al. TET2-interacting long noncoding RNA promotes active DNA demethylation of the MMP-9 promoter in diabetic wound healing. Cell Death Dis. 2019;10(11):813. doi:10.1038/s41419-019-2047-6

99. Alitalo K, Tammela T, Petrova TV. Lymphangiogenesis in development and human disease. Nature. 2005;438(7070):946-953. doi:10.1038/nature04480

100. Maruyama K, Asai J, Ii M, et al. Decreased macrophage number and activation lead to reduced lymphatic vessel formation and contribute to impaired diabetic wound healing. Am J Pathol. 2007;170(4):1178-1191. doi:10.2353/ajpath.2007.060018

101. He Z, Wei T, Zhang P, et al. Long noncoding RNA-antisense noncoding RNA in the INK4 locus accelerates wound healing in diabetes by promoting lymphangiogenesis via regulating miR-181a/Prox1 axis. J Cell Physiol. 2019;234(4):4627-4640. doi:10.1002/jep.27260
102. Tammela T, Alitalo K. Lymphangiogenesis: molecular mechanisms and future promise. Cell. 2010;140(4):460-476. doi:10.1016/j.cell.2010.01.045

103. Li B, Zhou Y, Chen J, et al. Long noncoding RNA H19 acts as a miR-29b sponge to promote wound healing in diabetic foot ulcer. FASEB J. 2021;35(1):e20526. doi:10.1096/ fj.201900076RRRRR

104. Merk DR, Chin JT, Dake BA, et al. MiR-29b participates in early aneurysm development in Marfan syndrome. Circ Res. 2012;110 (2):312-324. doi:10.1161/CIRCRESAHA.111.253740

105. $\mathrm{Hu} \mathrm{M}, \mathrm{Wu} \mathrm{Y}$, Yang $\mathrm{C}$, et al. Novel long noncoding RNA lnc-URIDS delays diabetic wound healing by targeting Plod1. Diabetes. 2020;69(10):2144-2156. doi:10.2337/db20-0147

106. Myllyharju J, Kivirikko KI. Collagens, modifying enzymes and their mutations in humans, flies and worms. Trends Genet. 2004;20(1):33-43. doi:10.1016/j.tig.2003.11.004

\section{Publish your work in this journal}

Diabetes, Metabolic Syndrome and Obesity: Targets and Therapy is an international, peer-reviewed open-access journal committed to the rapid publication of the latest laboratory and clinical findings in the fields of diabetes, metabolic syndrome and obesity research. Original research, review, case reports, hypothesis formation, expert opinion and commentaries are all considered for publication. The manuscript management system is completely online and includes a very quick and fair peer-review system, which is all easy to use. Visit http://www.dovepress.com/testimonials.php to read real quotes from published authors. 